ARTICLE

Received 7 Mar 2014 | Accepted 18 Sep 2014 | Published 12 Nov $2014 \quad$ DOl: 10.1038/ncomms6312

\title{
PP2A and Aurora differentially modify Cdc13 to promote telomerase release from telomeres at $\mathrm{G} 2 / \mathrm{M}$ phase
}

Zih-Jie Shen¹, Pang-Hung Hsu²,3, Yu-Tai Su¹, Chia-Wei Yang¹, Li Kao', Shun-Fu Tseng1, Ming-Daw Tsai ${ }^{2,4} \&$ Shu-Chun Teng ${ }^{1}$

In yeast, the initiation of telomere replication at the late $\mathrm{S}$ phase involves in combined actions of kinases on $\mathrm{Cdc} 13$, the telomere binding protein. $\mathrm{Cdc} 13$ recruits telomerase to telomeres through its interaction with Est1, a component of telomerase. However, how cells terminate the function of telomerase at $G 2 / M$ is still elusive. Here we show that the protein phosphatase 2A (PP2A) subunit Pph22 and the yeast Aurora kinase homologue Ipl1 coordinately inhibit telomerase at $\mathrm{G} 2 / \mathrm{M}$ by dephosphorylating and phosphorylating the telomerase recruitment domain of $\mathrm{Cdc13}$, respectively. While Pph22 removes Tel1/Mec1-mediated Cdc13 phosphorylation to reduce Cdc13-Est1 interaction, Ipl1-dependent $\mathrm{Cdc13}$ phosphorylation elicits dissociation of Est1-TLC1, the template RNA component of telomerase. Failure of these regulations prevents telomerase from departing telomeres, causing perturbed telomere lengthening and prolonged $\mathrm{M}$ phase. Together our results demonstrate that differential and additive actions of PP2A and Aurora on Cdc13 limit telomerase action by removing active telomerase from telomeres at G2/M phase.

\footnotetext{
${ }^{1}$ Department of Microbiology, College of Medicine, National Taiwan University, Taipei 10051, Taiwan. ${ }^{2}$ Genomics Research Center, Academia Sinica, Taipe 11529, Taiwan. ${ }^{3}$ Institute of Bioscience and Biotechnology, National Taiwan Ocean University, Keelung 20224, Taiwan. ${ }^{4}$ Institute of Biological Chemistry, Academia Sinica, Taipei 11529, Taiwan. Correspondence and requests for materials should be addressed to S.-C.T. (email: shuchunteng@ntu.edu.tw).
} 
T elomeres are dynamic DNA-protein complexes that protect the ends of linear chromosomes, prevent detrimental chromosome rearrangements and defend against genomic instability and the associated risk of cancer ${ }^{1-5}$. Telomeres, consisting of tandem repeats of short G-rich sequences, are synthesized by the enzyme telomerase ${ }^{6,7}$. The catalytic core of telomerase is composed of a reverse transcriptase and an RNA subunit. The reverse transcriptase utilizes the RNA component as a template to add the G-rich repeats onto the $3^{\prime}$ ends of the chromosome ${ }^{6-8}$.

In budding yeast Saccharomyces cerevisiae, genes encoding components of telomerase have been identified and mutations in these genes cause a gradual loss of telomere length ${ }^{9,10}$. EST2 and TLC1 encode the reverse transcriptase catalytic protein subunit and the templating RNA, respectively ${ }^{9,11,12}$. In addition, the protein encoded by EST1 is associated with the RNA component of telomerase, TLC1 (refs 13-16). Other accessory factors, such as $\mathrm{Cdc13}$, are required for the in vivo action of telomerase. $\mathrm{Cdc13}$ is a single-stranded telomere-binding protein ${ }^{17,18}$, which forms a complex with Stn1 and Ten1. This replication protein A-like heterotrimeric complex ${ }^{19}$ specifically binds single-strand telomeric sequences and is required for both telomere protection and telomerase recruitment ${ }^{17,18,20}$. It recruits telomerase to its site of action through an electrostatic interaction between Cdc13 and Est1 (refs 20,21). Some recent studies have suggested that Cdk1, Tell and Mecl may phosphorylate the telomerase recruitment domain of $\mathrm{Cdc13}$ to promote this Cdc13-Est1 interaction at the late $\mathrm{S}$ phase ${ }^{22-27}$.

Most somatic cells exit G1 to stay in quiescent stage, while cancer cells actively undergo cell cycles. Cell cycle is controlled by numerous mechanisms to ensure precise DNA replication and cell division. G2/M is the stage to terminate DNA replication and execute cell division. CDK, Aurora and Polo-like kinases are three major kinases to coordinate G2/M phase progression ${ }^{28}$. Aurora kinases are master controllers that manage multiple processes during cell division ${ }^{29}$. These mitotic Ser/Thr protein kinases mediate cellular reorganization through a spatially and temporally confined pattern of phosphorylation ${ }^{30}$. On the other hand, mitotic exit regulation requires inactivation of mitotic kinases and activation of counteracting protein phosphatases. Protein phosphatase 2A (PP2A) phosphatases are abundant in cells and are involved in many processes, including cell growth, differentiation, apoptosis, cell motility, DNA damage response and cell cycle progression ${ }^{31}$. It is capable of reversing the effects of CDK-mediated positive regulation through dephosphorylating Cdc25 and Wee1 (refs 32,33). In addition, PP2A is particularly important for timely dephosphorylation of various $\mathrm{CDK}$ substrates during mitotic exit ${ }^{34}$. PP2A, thus, opposes not only CDK activation but also its activity by dephosphorylating downstream effectors to promote efficient cell cycle progression.

Cdc13 binds Est1 to recruit telomerase ${ }^{35-37}$, and recent reports have suggested that the phosphorylations on the telomerase recruitment domain of $\mathrm{Cdc13}$ may promote telomerase recruitment ${ }^{22,26,27}$. However, this proposed signalling has been controversial $^{38,39}$. Here, we investigated the effects of Cdc13 phosphorylation on the Cdc13-Est1-telomerase signalling. We provided further evidence that phosphorylations of S249 and S255 of Cdc13 contribute to Est1-mediated telomerase recruitment at the late S phase. Phosphorylated S249 and S255 of $\mathrm{Cdc13}$ are subsequently dephosphorylated by PP2A phosphatase and $\mathrm{Cdc} 13$ is phosphorylated by Aurora kinase at S314. Inactivation of these two regulations delays telomerase departure at the G2/M phase, which causes elongated telomeres and prolonged $\mathrm{M}$ phase. Interestingly, PP2A phosphatase and Aurora kinase use distinct mechanisms to remove telomerase from telomeres: while PP2A phosphatase alleviates the Cdc13-Est1 interaction, Aurora kinase reduces the Est1-TLC1 interaction. Therefore, cells use multiple pathways to differentially and additively remove telomerase from telomeres at the G2/M phase to terminate telomere replication.

\section{Results}

PP2A dephosphorylates Cdc13 serine 249 and 255. Previous studies imply that phosphorylation of Cdc13 may promote telomerase recruitment (Fig. 1a) ${ }^{22-27}$. To examine the possibility that recruitment of telomerase by $\mathrm{Cdc} 13$ may be repressed by phosphatases, we determined the phosphorylation level of Cdc13 throughout the cell cycle for wild type (WT) and various phosphatase deletion strains. As shown in Fig. $1 \mathrm{~b}$ and Supplementary Fig. 1, WT Cdc13 is hyper-phosphorylated at late $S$ phase (40 $\mathrm{min})$ and then decreased at G2/M (60-140 min) after $\alpha$-factor synchronization. If Cdc13 is dephosphorylated by a phosphatase, phosphorylations should accumulate at the G2/M phase when the relevant phosphatase is eliminated. Some Tel1/ Mec1 substrates were previously reported to be removed by the phosphatases Pph3, Pph21, Pph22, Ptc2 and Ptc3 (ref. 40). We therefore tested the Cdc13 protein mobility in these corresponding deletion strains by western blot analysis. As shown at the lower panel of Fig. 1b, Cdc13 in the pph22 strain displayed a higher mobility shift than that in WT and other phosphatase mutant strains at the $60 \mathrm{~min}$ time points. The deletions did not significantly perturb the steady-state levels of Cdc13 (Fig. 1c). These results suggest that Pph22, a catalytic subunit of PP2A, may regulate the function of Cdc13.

Our previous study showed that $\mathrm{Cdc13}$ is phosphorylated at S225, S249, S255 and S306 by Tel1 and Mec1, and that S249 and S255 are two important residues to promote telomerase recruitment ${ }^{22}$. To test whether Pph22 targets these two phosphorylation sites, we raised a Cdc13 S249/S255 phosphospecific polyclonal antibody and the antibody exhibited higher binding affinity to phospho-S249/S255 and phospho-S255 than phospho-S249 (Supplementary Figs 2a, b and d). Meanwhile, this phospho-specific antibody could detect slight phosphorylation of Cdc13-S249/S255 in cdc13-Q250/Q256A cells, suggesting that other kinases might phosphorylate Cdc13-S249/S255 (Supplementary Fig. 2c). As shown in Fig. 1d, this antibody could recognize endogenously expressed WT Cdc13-Myc 9 protein immunoprecipitated from nocodazole-arrested yeast cells (lane 1). Reduction of phosphorylation was clearly observed in mec1, tel1 and mec1 tel1 mutant strains (lanes 2-4). Importantly, phosphorylation on S249/S255 accumulates in the G2/M arrested pph22 mutant (Fig. 1e). These data demonstrate that Pph22 can antagonize Tel1/Mec1-catalysed Cdc13 phosphorylation at $\mathrm{pS} 249 / \mathrm{pS} 255$ in vivo.

To determine whether Pph22 can directly regulate $\mathrm{Cdc13}$ phosphorylation, we phosphorylated recombinant Cdc13(227277), Cdc13-S249A(227-277) and Cdc13-S255A(227-277) by immunoprecipitated Mec1 and performed phosphatase assays with IgG-precipitated Pph22-TAP. Three IgG-precipitated tandem affinity purification (TAP) tagged proteins, Vps74, Pph3 and Pph21, were used as controls. Vps74 is required for Golgi localization of glycosyltransferases ${ }^{41}$; Pph3 was reported to dephosphorylate Cdc13 pS306 (ref. 42); and Pph21 is another nuclear phosphatase in the PP2A complex ${ }^{43}$. As shown in Fig. If, Pph21 and Pph22 efficiently dephosphorylated Cdc13 on single phosphorylated S249 or S255, or double S249/255 phosphorylations in vitro. Pph3 only dephosphorylated phosphorylated S249, whereas Vps74 showed no phosphatase activity on Cdc13 S249/S255 phosphorylations. Therefore, in vivo and in vitro data both demonstrate that Pph22 can dephosphorylate Cdc13 at S249 and S255. 


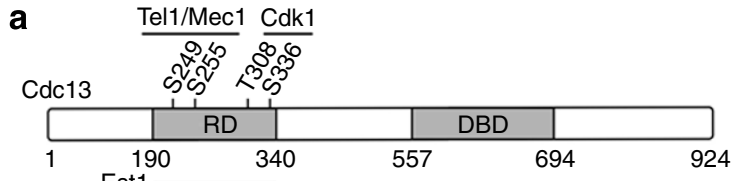

b
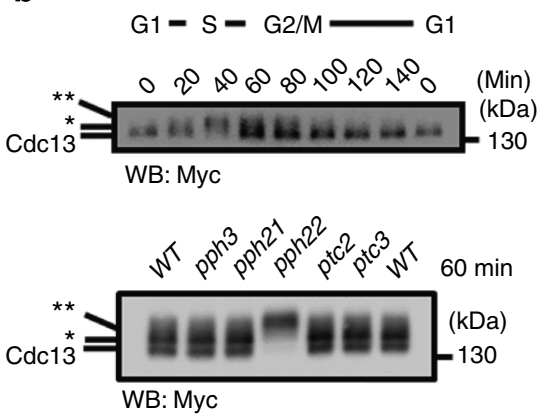

C

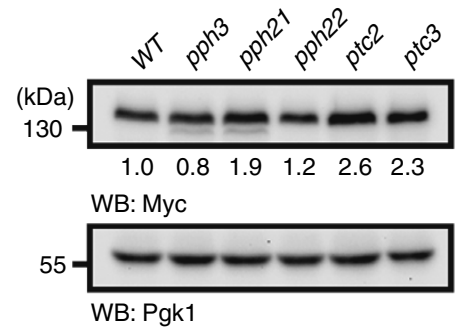

d

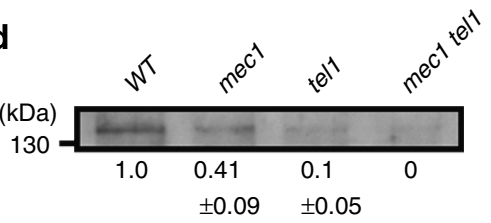

WB: Cdc13 pS249/pS255

$130-2=2$ e

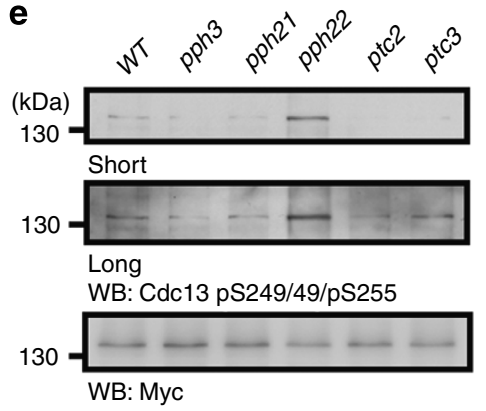

f

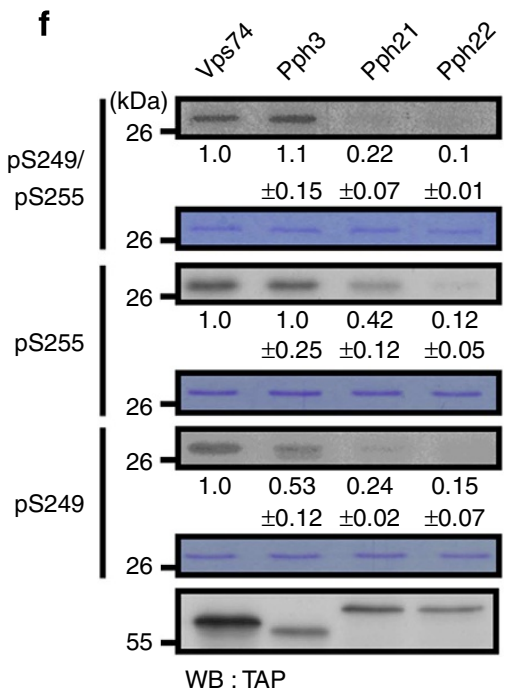

Figure 1 | Pph22 dephosphorylates phosphorylation of Cdc13 at Serine $\mathbf{2 4 9}$ and $\mathbf{2 5 5}$ in vivo and in vitro. (a) Schematic diagram of Cdc13 illustrates its domain structure, phosphorylation sites and kinases that phosphorylate these sites. The telomerase recruitment domain (RD) and DNA-binding domain (DBD) are indicated. (b) Cell cycle-dependent phosphorylation of Cdc13. Overnight culture was grown to early log phase in YPD, arrested at G1 by $\alpha$-factor, and released into cell cycle. Cells were collected at 20-min intervals for 140 min and Cdc13 was analysed by western blot analysis (WB). Cdc13 mobility in WT and different mutant strains was specifically compared at 60 and 80 min. SDS-PAGE (5\%) was used to separate the hyper- and hypo-phosphorylated $\mathrm{Cdc13}$, which are indicated by double $\left(^{\star \star}\right)$ and single asterisk $\left({ }^{\star}\right)$, respectively. (c) The steady-state levels of Cdc13 are not significantly affected in different phosphatase mutants. Endogenously expressed Cdc13 was chromosomally tagged with Myc $\mathrm{C}_{9}$. Pgk1 is a loading control. (d) Endogenously expressed $\mathrm{Cdc} 13$ was chromosomally tagged with $\mathrm{Myc}_{9}$. Cells were nocodazole-arrested and Cdc13 was immunoprecipitated. The pS249/pS255 phospho-specific antibody detected phosphorylations were decreased in mec1, tel1 and mec1 tel1 mutants. (e) Western analysis of immunoprecipitated Cdc13 from nocodazole-arrested phosphatase mutants using the pS249/pS255 phospho-specific antibody. Both long and short exposures are shown. (f) In vitro phosphatase assay. Recombinant Cdc13(227-277), Cdc13-S249A(227-277) and Cdc13-S255A(227-277) were ${ }^{32}$ P-labelled at S249 and S255 by Mec1. These substrates were treated with immunoprecipitants from the indicated tagged phosphatase strains. A non-phosphatase protein, Vps74, was used as a negative control. Proteins were resolved by SDS-PAGE and the phosphorylated proteins were detected by autoradiography (shown on top). The gels were also Coomassie blue stained (shown below) to confirm that samples were equally loaded.

Loss of $\mathrm{PPH} 22$ leads to delayed telomerase departure. To examine how telomeric functions are affected by Pph22, we analysed the effect of $p p h 22$ mutation on telomere replication. Heterozygous $p$ ph3/PPH3, pph21/PPH21 and pph22/PPH22 diploid strains (YPH501 background) were sporulated and dissected. The telomere length of each individual yeast colony derived from the dissected spores was measured after $\sim 100$ population doublings. Southern blot analysis showed that $p p h 22$, but not $p p h 3$ and $p p h 21$, mutation resulted in telomere lengthening (Fig. 2a), and this Pph22-mediated regulation was telomerase- and Tel1/Mec1 dependent (Supplementary Fig. 3), suggesting that Tel1/Mec1 and Pph22 participate in the same pathway. Telomere shortening was observed in $c d c 13-S 249 A$, $c d c 13-S 255 A$ and $c d c 13-S 249 / S 255 A$ cells from solid plates (Fig. 2b,c). In a liquid culture assay, when cultures starting from freshly dissected spores were repeatedly diluted, telomere shortening and marked telomere lengthening from survivor formation could be observed in telomerase pathway-defective strains after several dilutions ${ }^{44}$. Similarly, $c d c 13-S 249 / 255 A$ cells displayed gradual telomere shortening, survivor formation and cellular senescence phenotypes, and these phenotypes were recovered in cdc13-S249/255D cells (Supplementary Fig. 4). All these findings 
a

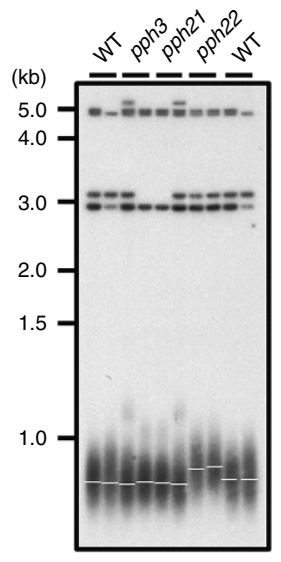

b

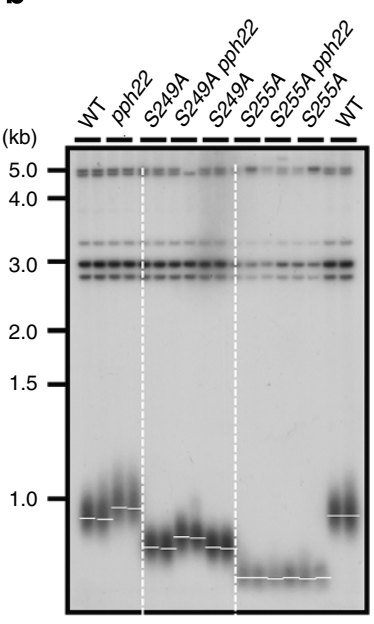

C

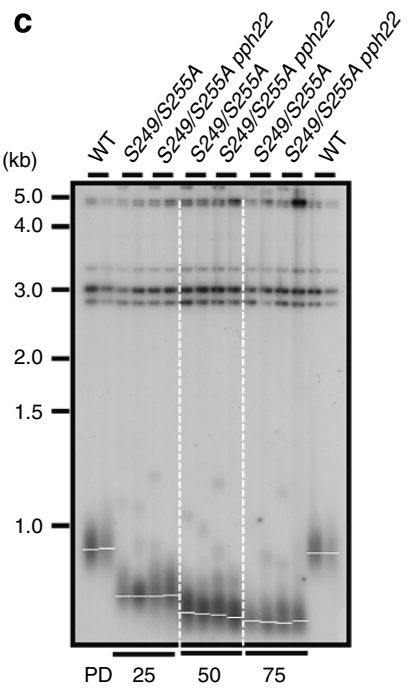

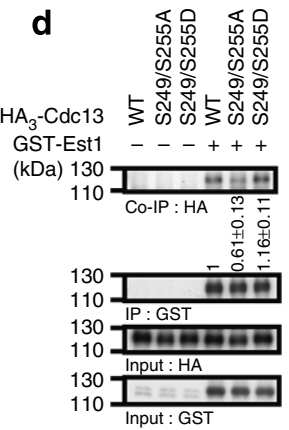

e

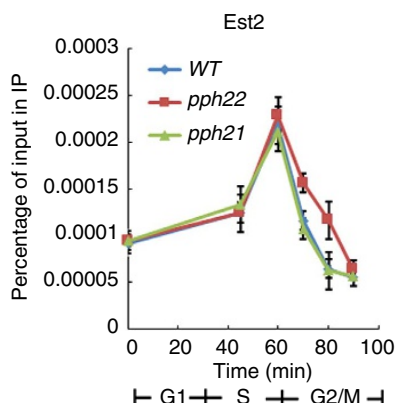

f

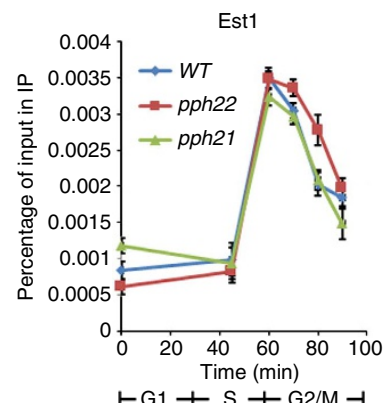

$-\mathrm{G} 1+\mathrm{s}+\mathrm{G} / \mathrm{M}-1$ g

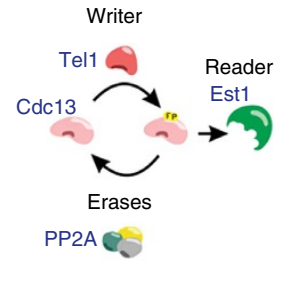

Figure 2 | pph22 mutation results in telomere lengthening and delayed eviction of telomerase. (a) Telomere analysis of pph21 and pph22 mutants. The telomere length of each individual colony derived from the dissected spore was measured at 100 generations (population doubling, PD100). (b) Telomere analysis of pph22 in WT, cdc13-S249A and cdc13-S255A mutation backgrounds. (c) Telomere analysis of pph22 in cdc13-S249/S255A background. Telomere length was measured at 25, 50 and 75 generations. (d) Co-IP assay indicated that cdc13-S249/S255A mutation reduces its interaction with Est1, whereas cdc13-S249/S255D increases. The levels of signal compared with that of the WT are shown below. (e) ChIP assays showed that cell cycle-dependent departure of Est2 at telomeres is delayed in pph22 mutant compared with that in WT and pph21 cells expressing Myc 18 -tagged Est2 ( $n=6, P$ values at 70 and $80 \mathrm{~min}$ are $<0.01$, Student's t-test, two tailed). Bars, s.d. (f) ChIP assays showed that cell cycle-dependent departure of Est1 at telomeres is delayed in pph22 mutant compared with that in WT and pph21 cells expressing Myc 18 -tagged Est1 ( $n=6, P$ values at 70 and 80 min are $<0.01$, Student's t-test, two tailed). Bars, s.d. (g) The stepwise modification on Cdc13 provides a writer-reader-eraser circuit to govern telomerase recruitment and departure. A white line marks the mean density of the autoradiographical signal in a-c.

indicate that phosphorylation/dephosphorylation of these two residues modulate telomerases recruitment. To further investigate whether one dephosphorylation site is more critical than the other in the Pph22-mediated function, the telomere length was analysed in spores from diploid yeast strains harbouring $p p h 22$ in combination with $c d c 13-S 249 A, c d c 13-S 255 A$ or $c d c 13-S 249 /$ S255A. Telomere was longer in pph22 and pph22 cdc13-S249A strains than that in WT and $c d c 13-S 249 A$, respectively (Fig. 2b,c and Supplementary Fig. 5), but these phenotypes were eliminated in $c d c 13-S 255 A$ and $c d c 13-S 249 / S 255 A$. Therefore, we conclude that dephosphorylation at Cdc13 S255 is more critical for Pph22mediated regulation. Finally, we wish to address whether Pph22mediated Cdc13 dephosphorylation is essential for de novo telomere addition. We tested the sudden telomere elongation by the de novo telomere addition assay (Supplementary Fig. $6 \mathrm{a})^{45}$. Cells that were arrested by $\alpha$-factor at G1 phase were released into the cell cycle and the pph22 strain displayed faster kinetics of telomere addition than WT and pph21 cells in the subsequent generations (Supplementary Fig. 6b). This result indicates that dephosphorylation of $\mathrm{Cdc1} 3$ at amino acids 249 and 255 reduces de novo telomere addition.
Cdc13 recruits telomerase mainly through binding to a telomerase subunit Est1 to recruit the RNA subunit of telomerase, TLC1 (ref. 35). Since S249 and S255 lie in the Est1-interacting region $^{20,36,37}$, we next asked whether Tel1/Mec1/Pph22dependent phosphorylation/dephosphorylation of $\mathrm{Cdc13}$ plays a role in coordinating the interaction between $\mathrm{Cdc13}$ and Est1. To this end, the chromosomal copy of CDC13 and EST1 was tagged with hemagglutinin $\left(\mathrm{HA}_{3}\right)$ and glutatione S-transferase (GST), respectively, and expressed under the control of galactoseinducible promoters. Overexpressed GST-Est1 was precipitated by anti-GST antibodies and precipitates were detected by western blot analysis for $\mathrm{HA}_{3}-\mathrm{Cdc13}$. We first verified the interaction between Cdc13 and Est1 (Fig. 2d, lanes 1 and 4). Mutation of S249 and S255 to alanine decreased the interaction between Cdc13 and Est1 (lane 5) but mutation to phosphomimetic aspartic acid maintained the interaction (lane 6). Chromatin immunoprecipitation (ChIP) assays showed that cell cycledependent departures of Est2 and Est1 from telomeres were also delayed in yeast harbouring pph22 mutant compared with those in WT and pph21 strains (Fig. 2e,f and Supplementary Figs 7 and 8). Since $c d c 13-S 255 A$ mutation caused a more 
prominent effect on telomere shortening, we examined whether $c d c 13-S 255 A$ mutation abolished the recruitment of telomerase (Supplementary Fig. 9). Cell cycle-dependent recruitment of Est2 and Est1 was compromised in $c d c 13-S 255 A$ and $c d c 13-S 255 A$ pph22 cells, suggesting that the S255 phosphorylation plays a critical role in telomerase recruitment. Together, these data indicate that Pph22-dependent dephosphorylation of $\mathrm{Cdc} 13$ provides a negative regulation on the Cdc13-Est1 interaction and telomerase recruitment during cell cycle progression (Fig. 2g).

Aurora phosphorylates Cdc13 serine 314 at G2/M phase. After obtaining the above results, we wondered whether there are additional critical regulations that might be essential to stop telomere replication at the G2/M phase. Since multiple phosphorylations on $\mathrm{Cdc} 13$ promote telomerase recruitment, we surmised that distinct $\mathrm{Cdc13}$ phosphorylations may also modulate telomerase departure. Using mass spectrometry (MS) to analyse phosphorylation sites of overexpressed Cdc13- $\mathrm{Myc}_{9}$ in S. cerevisiae, we identified new phosphorylation sites S314, S324 and S333 on $\mathrm{Cdc13}$ (Fig. 3a) that have not been well studied previously $22,26,27,42$ (Supplementary Fig. 10). We then used Southern blot analysis to examine whether these phosphorylations would affect telomere replication (Fig. 3b). Interestingly, an apparent telomere lengthening was observed in $c d c 13-S 314 A$ cells. By bioinformatic analysis, we found that $\mathrm{S} 314$ fits into a consensus sequence of Aurora kinase substrates (Fig. $3 c)^{46}$. We thus raised a pS314-specific antibody to examine $\mathrm{Cdc13}$ phosphorylation and its regulation in vivo. Western blot analysis of immunoprecipitated Cdc13-Myc 9 showed that this antibody was phospho-specific and the antibody recognized signal was abolished when immunoprecipitants was prepared from the S314A mutant (Supplementary Fig. 11). Phosphorylation of S314 was largely dependent on Aurora kinase because the signal was greatly reduced in immunoprecipitants prepared from nocodazole-arrested temperature-sensitive ipl1-4 mutant following growth at non-permissive temperature (Fig. 3c). To identify at which cell cycle stage $\mathrm{Cdc13}$ is phosphorylated, cultures synchronized with $\alpha$-factor were analysed by phosphospecific antibodies. As shown in Fig. 3d, phosphorylation of S249 and S255 was detected at $40 \mathrm{~min}$ after release in WT but the signal accumulated from 60 to $100 \mathrm{~min}$ in the pph22 mutant strain. On the other hand, phosphorylation of S314 accumulated after $60 \mathrm{~min}$ and reached maximum at G1. Immunoprecipitation (IP) kinase assay further demonstrated that Ipl1 phoshporylates $\mathrm{Cdc13}$ in vitro using recombinant Cdc13 (Fig. 3e). These data suggest that Ipl1 directly phosphorylates Cdc13.



b

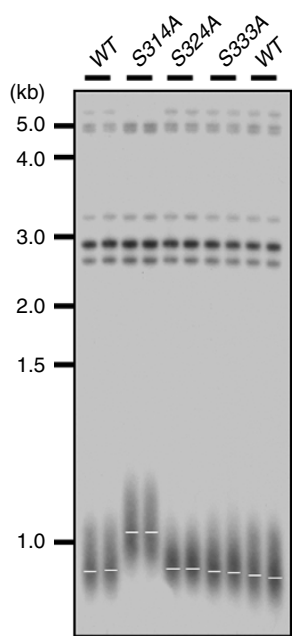

C

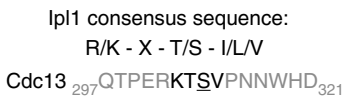

Ipl1 consensus sequence: $\mathrm{R} / \mathrm{K}-\mathrm{X}-\mathrm{T} / \mathrm{S}-\mathrm{I} / \mathrm{L} / \mathrm{V}$ Cdc13 $_{297}$ QTPERKTSVPNNWHD 321

d

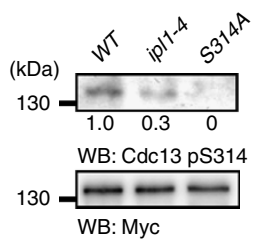

e


Figure 3 | Ipl1-mediated Cdc13 phosphorylation on S314 at M phase. (a) Schematic diagram of Cdc13 illustrates its domain structure and newly identified phosphorylation sites. The recruitment domain (RD) and DNA-binding domain (DBD) domains are indicated. (b) Telomere analysis of newly identified phosphorylation sites of $\mathrm{Cdc13}$. The telomere length of individual colony derived from the dissected spore was measured at 100 population doubling (PD100). A white line marks the mean density of the autoradiographical signal. (c) The phosphorylation site of Cdc13 S314 fits into the Ipl1 consensus site $\left(\mathrm{R} / \mathrm{K}-\mathrm{X}-\mathrm{T} / \mathrm{S}^{\star}-\mathrm{I} / \mathrm{L} / \mathrm{V}\right)^{46}$. Endogenously expressed $\mathrm{Cdc} 13$ was chromosomally tagged with $\mathrm{Myc}$. Cells were arrested by nocodazole and cultures were shifted to the non-permissive temperature of the ip/1-4 strain $\left(37^{\circ} \mathrm{C}\right)$. The pS314 phospho-specific antibody detected immunoprecipitated WT but not Cdc13-S314A mutant protein, and the signal was decreased in temperature-sensitive ip/1-4 mutants. The same blot was stripped and reprobed with an anti-Myc antibody. (d) Phosphorylations of Cdc13 S249/S255 and S314 are cell cycle dependent. Phosphorylation of Cdc13 S249/S255 is enriched $\sim 40-60 \mathrm{~min}$ (late S/G2) after $\alpha$-factor synchronization and release. Phosphorylation of Cdc13 S314 is decreased from G1 to S phase, and enriched from M to $\mathrm{G} 1$ phase. $\alpha$-Factor was added at $60 \mathrm{~min}$ again to prevent cells re-entering the next cell cycle. The quantitative analysis is shown below. Bars, s.d. (e) In vitro kinase assay was performed using immunoprecipitants of Flag-tagged Ipl1 WT or kinase-dead mutant on recombinant Cdc13(276-332). Western blot analysis was conducted using Cdc13 pS314 phospho-specific or Flag antibodies. The kinase reactions were also Coomassie blue (CB) stained (shown below) to confirm that lanes were equally loaded. 
Given the fact that both S249/S255 and S314 are located at the telomerase recruitment domain, we wondered whether there is an interplay between phospho-S249/S255 and phospho-S314. Western blot analysis demonstrated that phosphorylations on S249 and S255 were only slightly altered in $c d c 13-S 314 A$ cells (Supplementary Fig. 11d). On the other hand, a 33\% reduction on S314 phosphorylation was observed in $c d c 13-S 249 / S 255 A$ cells (Supplementary Fig. 11d). These results indicate that the phosphate groups on S249 and S255 may have an influence on the Aurora kinase-mediated S314 phosphorylation.

To identify the potential cellular functions for Cdc13 S314 phosphorylation, we tested whether other telomere-related mutations would affect S314-mediated phenotypes. Telomere length was analysed in $c d c 13-S 314$ strains in combination with or without deletion of known factors involved in the telomerase pathway (Fig. $4 \mathrm{a}-\mathrm{c}$ and Supplementary Fig. 12). In WT and mec1 strains, single mutation serine to alanine caused telomere lengthening. These results indicate that the negative charge on this residue at a particular timing is necessary for telomere length homeostasis. Moreover, no additional or synergistic effect on telomere lengthening was observed in cdc13-S314 tel1, cdc13T308A-S314 and $c d c 13-S 314$ tlc1 strains. These data suggest that the S314 phosphorylation-mediated telomere regulation depends on telomerase. Besides, in the absence of Tel1/Cdk1-dependent phosphorylation on Cdc13, Ipl1-mediated phosphorylation on S314 does not modulate telomere replication.

Aurora accelerates eviction of telomerase at G2/M phase. Since serine 314 is located in the Est1- and Stn1-interacting region of Cdc13 (Fig. 3a) 20,36,37,47, we next asked whether Ipl1-dependent phosphorylation of $\mathrm{Cdc13}$ plays a role in coordinating the interaction of Cdc13 with Est1 or Stn1. When GST-Est1, GSTStn 1 and $\mathrm{HA}_{3}-\mathrm{Cdc} 13$ were overexpressed using galactoseinducible promoters, we detected the Cdc13-Est1 and Cdc13Stn1 interactions. Mutation of S314 to alanine or aspartic acid did not affect the interaction of $\mathrm{Cdc} 13$ with Est1 or Stn1 (Supplementary Fig. 13). Furthermore, mutation of S314 to alanine or aspartic acid did not affect the cell cycle-dependent departure of Est1 (Fig. 5a). However, cell cycle-dependent departure of Est2 from telomeres was delayed in yeast harbouring $c d c 13-S 314 A$ mutant compared with that in WT
(Fig. 5b and Supplementary Fig. 14). Mutation of S314 to alanine resulted in a twofold increase of TLC1 RNA coimmunoprecipitated with $\mathrm{Cdc} 13$ (Fig. 5c). Conversely, coimmunoprecipitated TLC1 was reduced in the $c d c 13-S 314 D$ mutant (Fig. 5c). The interaction between Est1 and TLC1 was increased in the $c d c 13-S 314 A$ mutant compared with that in WT (Fig. 5d), and co-immunoprecipitated TLC1 was reduced in the cdc13-S314D mutant (Fig. 5d). We could not detect a difference in Est2-TLC1 association in cdc13-S314 mutation background (Fig. 5e). On the other hand, cell cycle-dependent Cdc13/Est1mediated TLC1 association was increased in yeast harbouring cdc13-S314A mutant compared with that in WT (Fig. 5f and Supplementary Fig. 14). Together, these data indicate that Ipl1dependent phosphorylation of $\mathrm{Cdc} 13$ plays a role to telomerase RNA departure during cell cycle progression. This regulation on telomerase departure is not through perturbing the Cdc13-Est1 interaction, but through alleviating the Est1-TLC1 interaction.

PP2A and Aurora act independently but additively. Finally, we asked whether PP2A phosphatase and Aurora kinase work through independent pathways. Cell cycle progression and telomere length were examined in WT, single and double mutants. Additive effects in cell cycle delay and telomere lengthening were observed in the pph22 cdc13-S314A strain (Fig. 6a,b). These phenotypes were not observed in the pph22 ipl1-4 strain (Supplementary Fig. 15), probably because Ipl1 regulates too many downstream substrates and all these regulations may engender a combinatively dominant effect on telomeres. Cell cycle-dependent departure of Est2 from telomeres was further delayed in the $p p h 22 c d c 13-S 314 A$ double mutant compared with that in $p p h 22$ and in $c d c 13-S 314 A$ strains (Fig. $6 c$ and Supplementary Fig. 7), as observed that Est2 binding to telomeres was 70, 34 and 44 percentage higher at the 70-min time point in pph22 cdc13-S314A, pph22 and cdc13-S314A mutants, respectively, compared with that in the WT (Fig. 6c). Previous studies have shown that $\mathrm{G} 2 / \mathrm{M}$ delay alone is not sufficient to trigger telomere lengthening (Supplementary Table 1), indicating an active role of Pph22 and Ipl1 in removing telomerase. These results suggest that Pph22 and Ipl1 control telomere replication through different pathways and each contributes to telomerase departure.
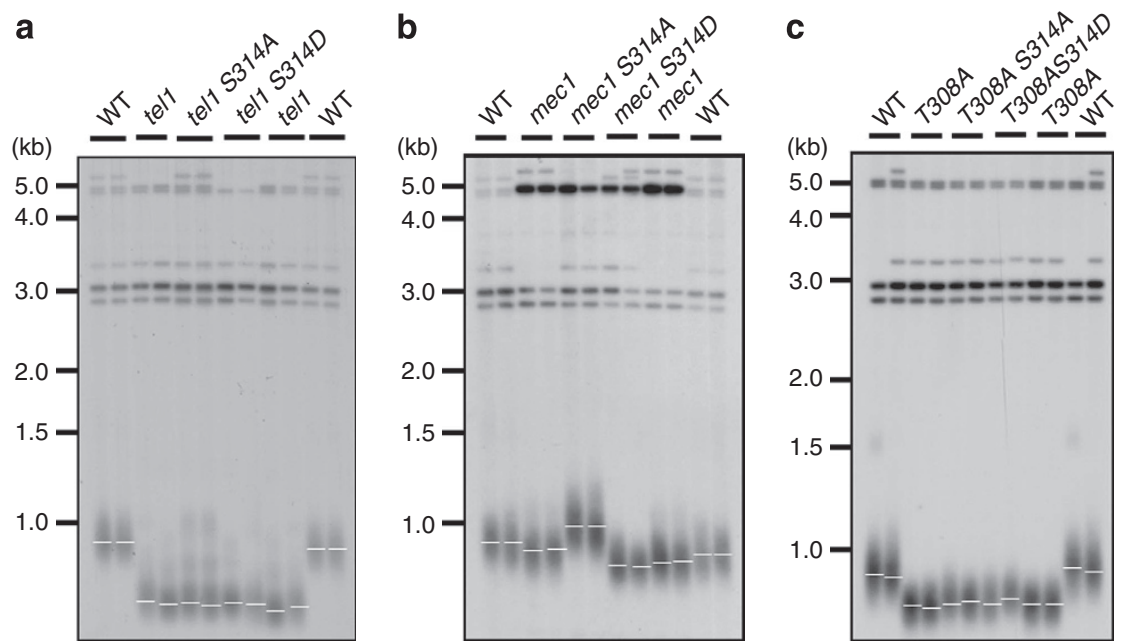

Figure 4 | Telomere length regulation by Cdc13 Serine 314 mutation is Tel1/Cdk1 dependent. (a) Telomere analysis of Cdc13 S314 in WT and tel1 backgrounds. (b) Telomere analysis of Cdc13 S314 in WT and mec1 backgrounds. A black line marks the mean density of the autoradiographical signal. (c) Telomere analysis of Cdc13 S314 in WT, and cdc13-T308A backgrounds. A black line marks the mean density of the autoradiographical signal. A black line marks the mean density of the autoradiographical signal in a-c. 
a

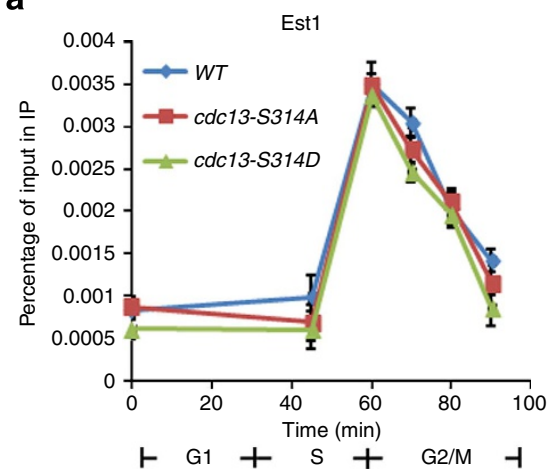

b
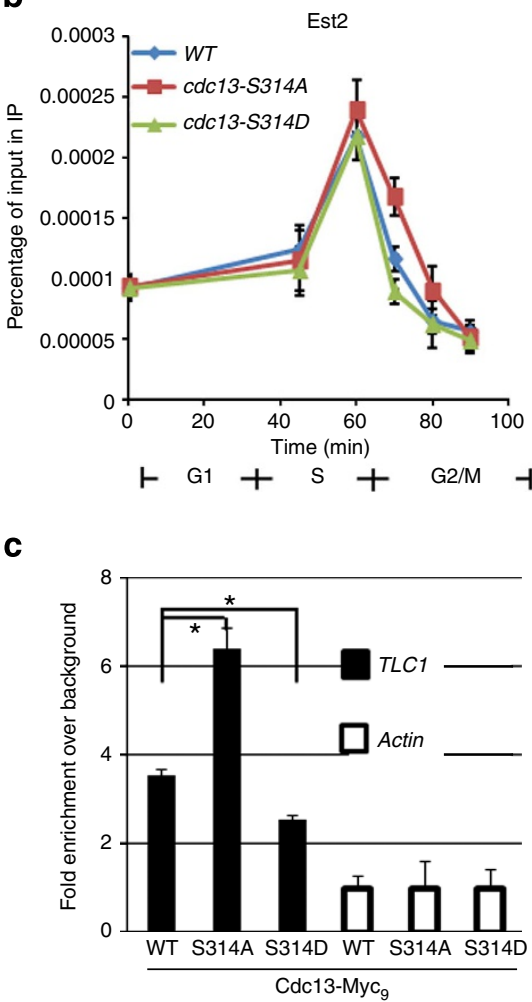

d

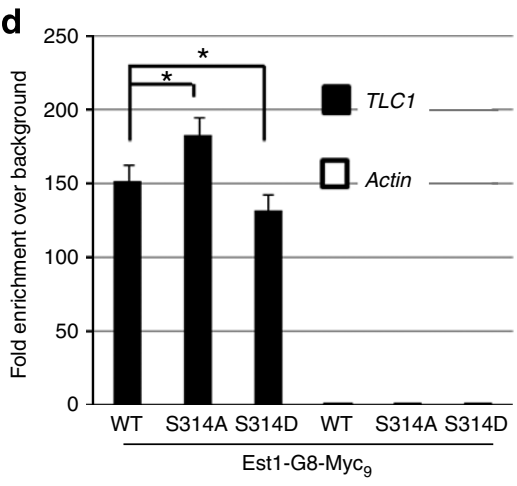

e

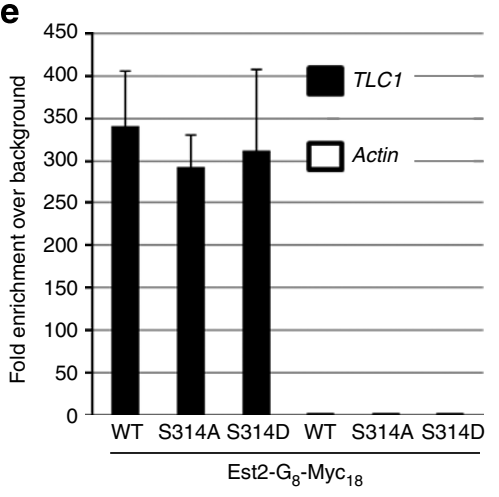

f

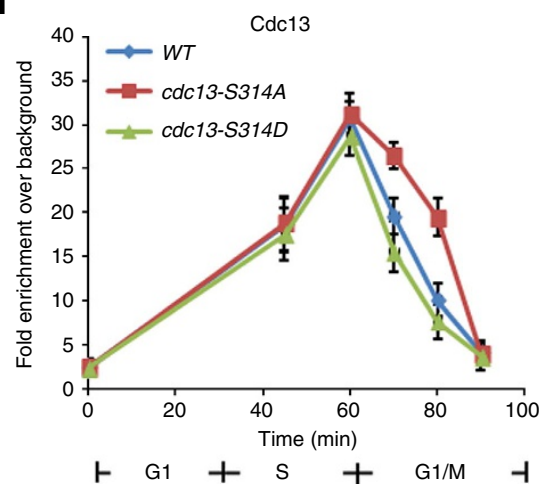

Figure 5 | $\mathbf{S 3 1 4}$ phosphorylation accelerates the departure of telomerase at $\mathbf{M}$ phase. (a) ChIP assays showed that cell cycle-dependent departure of Est1 at telomeres is not delayed in $c d c 13-S 314 A$ and in $c d c 13-S 314 D$ strains ( $n=6, P$ values at 70 and 80 min are $>0.05$, Student's $t$-test, two tailed). Bars, s.d. (b) ChIP assays showed that cell cycle-dependent departure of Est2 at telomeres is delayed in cdc13-S314A mutant compared with that in WT and cdc13-S314D cells expressing Myc 18 -tagged Est2 ( $n=6, P$ values at 70 and 80 min are $<0.01$, Student's t-test, two tailed). Bars, s.d.

(c) IP of Cdc13-Myc 9 from nocodazole-arrested culture in vivo. Co-immunoprecipitated TLC1 RNA was quantified by real-time reverse transcription PCR (RT-PCR). Telomerase recruitment efficiency is reduced in the $c d c 13-S 314 D$ strain $(n=6, P$ values $<0.01$, Student's $t$-test) and conversely increased in the $c d c 13-S 314 A$ strain $\left(n=6,{ }^{\star} P\right.$ values $<0.01$, Student's $t$-test). Bars, s.d. (d) IP of Est1-Myc 9 from nocodazole-arrested culture in vivo. Co-immunoprecipitated TLC1 RNA was quantified by real-time RT-PCR. Telomerase recruitment efficiency is reduced in the $c d c 13-S 314 D$ strain $(n=6$, $P$ values $<0.01$, Student's $t$-test) and conversely increased in the $c d c 13-S 314 A$ strain $\left(n=6,{ }^{\star} P\right.$ values $<0.01$, Student's $t$-test). Bars, s.d. (e) IP of Est2-Myc 18 in vivo. Co-immunoprecipitated TLC1 RNA was quantified by real-time RT-PCR. (f) IP of Cdc13-Myca through cell cycle progression in vivo. Co-immunoprecipitated TLC1 RNA was quantified by real-time RT-PCR. TLC1 binding to Cdc13 is increased in the $c d c 13-S 314 A$ strain $(n=6, P$ values at 70 and 80 min are $<0.01$, Student's t-test) Bars, s.d.

\section{Discussion}

We previously found that Tel1 and Mec1 phosphorylate Cdc13 at S249 and S255 in vitro 22 . Several laboratories also discovered genetic and physical evidence of the importance of Tel1 in telomerase recruitment ${ }^{23-25}$. In this study, our phospho-specific antibody detected in vivo S249 and S255 phosphorylation in a Tel1/Mec1-dependent and cell cycle-dependent manner and the signal was abolished in the $c d c 13$ S249S255A cells. Moreover, gradual telomere shortening and cellular senescence were observed in $c d c 13-S 249 / S 255 A$ cells, but partly recovered in cdc13-S249/S255D cells. All these findings suggest that the negative charges at the telomerase recruitment domain of Cdc13 facilitate the Cdc13-Est1 salt-bridge formation ${ }^{26,48}$. Contrary to expectations, a two-hybrid assay ${ }^{38}$ found that the 
a

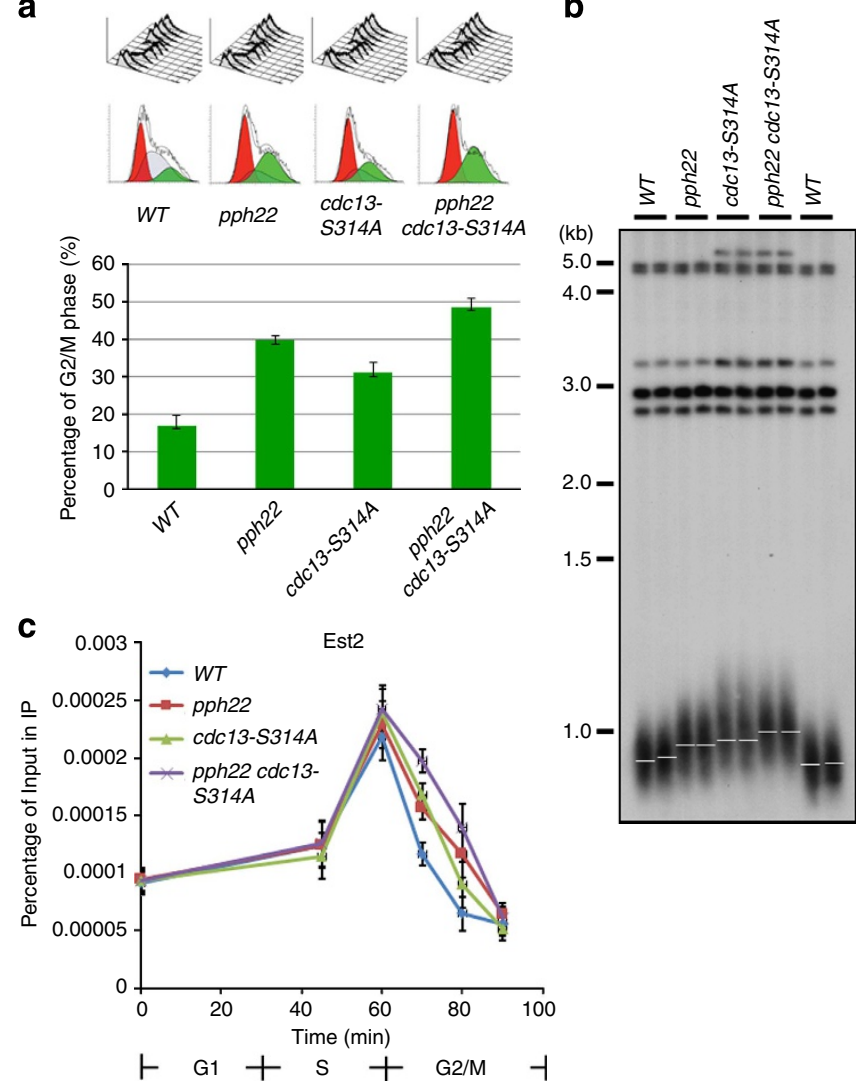

Figure 6 | PP2A phosphatase and Aurora kinase independently contribute to telomerase departure. (a) Delay of cell cycle in pph22 cdc13S314A cells. Cells were $\alpha$-factor synchronized and released at $30^{\circ} \mathrm{C}$. Aliquots were taken at indicated time points for flow cytometry analysis. The data at $120 \mathrm{~min}$ are specifically shown. Percentages of cell cycle stages were quantified according to the manufactures instructions (Becton Dickinson). Bars, s.d. (b) Telomere analysis of pph22 in the WT or cdc13S314A background. Cells used in this experiment were derived from the dissected spores at 100 population doubling (PD100). A white line marks the mean density of the autoradiographical signal. (c) ChIP assays showed that cell cycle-dependent departure of Est2 at telomeres is additively delayed in the pph22 cdc13-S314A strain compared with that in single mutants ( $n=6, P$ values at 70 is $<0.01, P$ values at 80 is $<0.05$, Student's $t$-test, two tailed). Bars, s.d.

Cdc13 and Est1 interaction is Tel1 independent. Lack of in vivo evidence of Cdc13 S249 and S255 phosphorylation previously has caused some reasonable speculation and debate in this field. However, all these arguments are understandable and explainable. For example, the interaction between $\mathrm{Cdc13}$ and Est1, as monitored by the two-hybrid assay, is S255 dependent but Tel1 independent ${ }^{38}$. In that case, two-hybrid assay might measure a basal level interaction between $\mathrm{Cdc13}$ and Est1, and phosphorylation may stimulate their interaction in vivo only at the late S phase. However, this Tel1-mediated enhancement may not be detectable owing to the excess amounts of $\mathrm{Cdc13}$ and Est1 throughout the whole-cell cycle in the two-hybrid system, which may be over the enzymatic loading for the endogenous Tel1 kinase. Here we present evidence for the first time that Cdc13 S249 and S255 are phosphorylated in vivo at the late S phase (Fig. 3c). Cdc13 S249 and S255 are partially phosphorylated in $c d c 13-Q 250 / Q 256 A$ mutant cells (Supplementary Fig. 2c), and the cdc13-Q250/Q256A mutation causes slight telomere shortening ${ }^{38}$. Therefore, we hypothesize that other protein kinases might phosphorylate Cdc13 S249 and S255, especially in the absence of Tel1/Mec1 kinases. The phosphorylation of Cdc13-S249/S255 is critical for telomerase recruitment and this action cannot be completely recovered by aspartate replacement in vivo. However, Cdc13-S249/S255D retains its ability to interact with Est1 in our in vitro co-IP assay. A possible explanation is that the S249/S255D mutation may bind to Est1 with the same affinity, but this binding leads to some allosteric effect on Est1TLC1 association. Alternatively, in vitro co-IP assay using overexpressed and tagged proteins may not completely reflect the exact affinity between $\mathrm{Cdc13}$ and Est1 in vivo, and Cdc13S249/S255D does contain a little defect in Est1 binding. Our findings further strengthens the detailed mechanism of Tel1/Cdc13/Est1-mediated telomerase recruitment in S. cerevisiae ${ }^{20,22,23,25}$. The collective functions of Ccq1, Tppland Pot1 in Schizosaccharomyces pombe are analogous to the telomeric role of $\mathrm{Cdc13}$ in S. cerevisiae ${ }^{49,50}$. Consistently, ataxia telangiectasia mutated (ATM)/ATM- and Rad3-related (ATR)-promoted Ccq1-Est1 interaction was recently revealed ${ }^{51}$. It will be interesting to know whether ATM/ATR promotes the TPP1-POT1 complex-mediated telomerase recruitment in mammals ${ }^{52,53}$ as observed in yeasts.

Adequate and timely regulation of DNA replication is critical for optimal progression of cell cycle. Telomere replication is facilitated by Cdk1/Tel1/Mec1-mediated Cdc13 phosphorylation at late $\mathrm{S}$ phase ${ }^{22-27}$. G2 phase is well known as the pre-mitotic phase to prepare for M phase entry. Another critical task at G2/M is to completely terminate DNA replication. Aurora is a master G2/M phase kinase ${ }^{28}$, and PP2A phosphatase counteracts ATM and ATR functions in DNA damage ${ }^{54}$. Here we demonstrate that both PP2A phosphatase and Aurora kinase promote the termination of telomere replication through modifications on Cdc13. A recent study also identifies that $\mathrm{S} 314$ of $\mathrm{Cdc13}$ is a phosphorylation site and this phosphorylation might provide a critical regulation in telomere replication ${ }^{55}$. It is interesting that PP2A phosphatase and Aurora kinase use distinct mechanisms to remove telomerase. PP2A phosphatase directly removes the phosphorylations on Cdc13 S249 and S255 to reduce the electrostatic interaction between $\mathrm{Cdc13}$ and Est1. On the other hand, Aurora kinase-mediated Cdc13 phosphorylation does not disturb the Cdc13-Est1 interaction but may lead to an allosteric modulation on Est1-TLC1 interaction that dissociates telomerase RNA. It may be possible that in a single cell, PP2A phosphatase helps to remove telomerase on some telomeres, whereas Aurora kinase promotes telomerase departure on other telomeres. Compromising PP2A phosphatase- and Aurora kinase-mediated regulations trigger unnecessary telomere elongation, which should be a waste of energy and lead to cell cycle delay. Moreover, although at G1 phase Aurora kinase-mediated S314 phosphorylation may provide an inhibitory effect on the Cdc13-Est1-TLC1 interaction, as previously suggested ${ }^{56}$ at G1 phase the telomerase complex may be further recruited by the Ku complex.

We previously identified that Cdk1-mediated Cdc13 phosphorylation also controls the stability of $\mathrm{Cdc13}$ (ref. 27). This kind of modulation may send a signal for the AAA ATPase Cdc48-facilitated $\mathrm{Cdc13}$ degradation ${ }^{57}$. Sequential phosphorylation and dephosphorylation on $\mathrm{Cdc13}$ therefore offer a great platform to modulate telomerase function in vivo. Moreover, Pif1 helicase is a negative regulator of telomere replication ${ }^{58}$. Its helicase function facilitates cells to remove telomerase RNA from telomeres ${ }^{59}$. All these regulations may additively contribute to telomerase departure and serve as gatekeepers to maintain efficient cell cycle (Fig. 7), and our findings provide evidence that not only initiation but also 


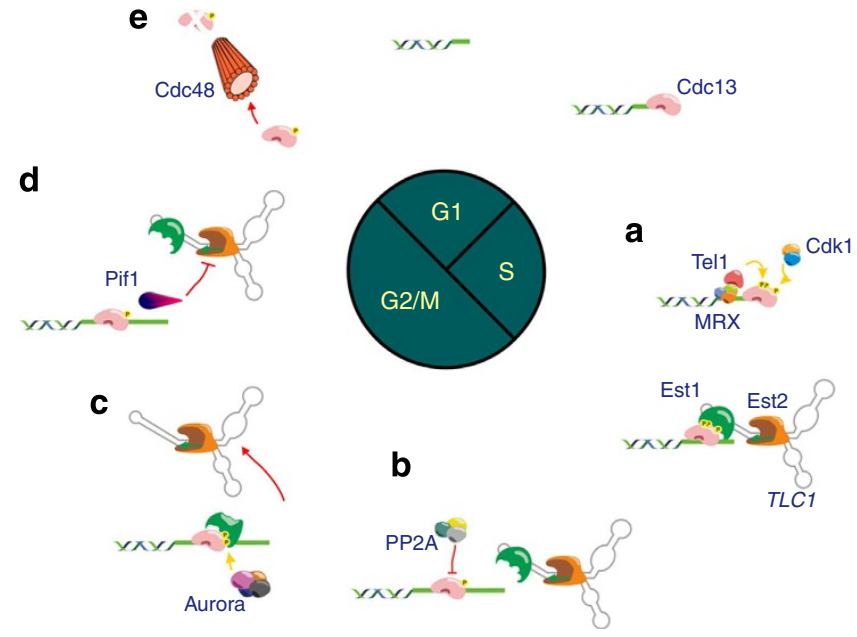

Figure 7 | Schematic model of PP2A phosphatase and Aurora kinasepromoted telomerase departure. (a) $\mathrm{Cdc} 13$ is phosphorylated by $\mathrm{Cdk} 1, \mathrm{Tel} 1$ and $\mathrm{Mec} 1$ at late $\mathrm{S}$ phase to recruit Est1 and the telomerase complex. (b) Pph22 dephosphorylates $\mathrm{Cdc} 13$ and dissociates Est1 to promote the departure of the telomerase complex. (c) Ipl1 phosphorylates $\mathrm{Cdc13}$ serine 314. It may lead to an allosteric modulation on Est1-TLC1 interaction that dissociates telomerase from telomeres. (d) Pif1 helicase removes telomerase RNA from telomeres ${ }^{59}$. (e) Cdk1 and AAA ATPase Cdc48 (ref. 57) facilitate $\mathrm{Cdc} 13$ degradation. Pathways b-d may occur on different telomeres to completely inactivate telomerase recruitment at $\mathrm{M}$ phase.

termination of telomerase recruitment plays an important role in telomere maintenance and cell cycle progression.

\section{Methods}

Strains and plasmids. All yeast operations were performed by standard methods ${ }^{60}$. Strains and plasmids used in this study are listed in Supplementary Tables 2 and 3. Mutant strains pph3A, pph21 4 and pph22 24 are isogenic to BY4741 (MATa his2 11 leu2 $\Delta 0$ met $15 \Delta 0$ ura3 $\Delta 0$ ) and were obtained from yeast deletion library (Invitrogen, 95401.H2). The yeast strains carrying tlc1, mec1, sml1, tel1, cdc13 and CDC13-Myc ${ }_{9}$ are isogenic to YPH499 (MATa ura3-52 lys2-801_amber ade2101_ochre trp1-463 his3-4200 leu2-41) (ref. 61). ipl1-4 is isogenic to Y300 (MATa trp1-1 ura3-1 his3-11,15 leu2-3,112 ade2-1 can1-100). PPH3-TAP, PPH21-TAP and PPH22-TAP are isogenic to W303 (MATa leu2-3,112 trp1-1 can1-100 ura3-1 ade2-1 his3-11,15). pRS304CDC13-Myc sas obtained from Dr Virginia Zakian $^{37}$ and pRS306CDC13 was constructed by PCR containing the CDC13 open read frame and the downstream 200 nucleotides. Point mutations were introduced into CDC13 using QuickChange site-directed mutagenesis (Stratagene). To generate chromosomal $c d c 13$ mutants, pRS306 cdc13 mutants were XhoI-digested and transformed into CDC13 strains, and the URA3 pop-out mutants were selected from the 5-fluoroorotic acid (5-FOA)-resistant colonies using PCR analysis. The ipl1-4 strain was constructed by a recombination-mediated two-step gene replacement procedure $^{62}$, replacing the IPL1 with the ipll-4 mutant allele of pCC321. The pph22::KanMX4 and pph22::HIS3 mutants were constructed by transforming pph22::KanMX4 and pph22::HIS3 PCR fragments, respectively, into the strains. Plasmids pGEX-4T-Cdc13(227-277) were constructed by ligating a PCR product containing amino acids 227-277 of Cdc13 from pRS314CDC13 into BamHI- and SalI-treated pGEX-4T. Plasmids pGEX-4T-Cdc13(276-332) were constructed by ligating a PCR product containing amino acids 276-332 of Cdc13 from pRS314CDC13 into BamHI- and XhoI-treated pGEX-4T. Plasmid pYES2-Cdc13$\mathrm{Myc}_{9}$ was constructed by ligation of a PCR product from $\mathrm{pRS} 314 \mathrm{Cdc} 13-\mathrm{Myc}_{9}$ into KpnI-Klenow-treated pYES2. All primer sequences for PCR and mutagenesis are provided in Supplementary Table 4 .

Gel electrophoresis and western blot analysis. Cells were grown at $30^{\circ} \mathrm{C}$ in yeast extract peptone dextrose (YPD) broth. Cell lysates were prepared with lysis buffer $(150 \mathrm{mM} \mathrm{NaCl}, 1 \%$ Nonidet P-40, $1 \%$ deoxycholate, $0.1 \%$ SDS, $50 \mathrm{mM}$ Tris$\mathrm{HCl}, \mathrm{pH} 7.5$, and protease inhibitors) or trichloroacetic acid (TCA) precipitated ${ }^{63}$. Whole proteins were extracted and resolved by $10 \%$ (except Fig. 1b) SDSpolyacrylamide gel electrophoresis (SDS-PAGE). For the supershift of Cdc13 phosphorylations, SDS-PAGE was performed by Bio-Rad PROTEIN II System. $\mathrm{Myc}_{9}$-tagged $\mathrm{Cdc} 13$ protein was detected by an anti-Myc antibody (Roche, working condition 1: 2,000). HA-tagged Cdc13 protein was detected by an anti-Myc antibody (Covance, working condition 1:2,000). GST-tagged overexpressed Cdc13, Est1 and Stn1 were detected by an anti-GST antibody (Roche, working condition 1:3,000). TAP-tagged phosphatase Pph3, Pph21 and Pph22 were detected by an anti-TAP antibody (Thermo Fisher Scientific, working condition 1:3,000). FLAGtagged Ipl1 was detected by an anti-FLAG antibody (Sigma, working condition 1:1,000). Affinity-purified rabbit anti-Cdc13 pS249/pS255 and pS314 phosphospecific antibodies (raised against phosphopeptides SGSGYIESpQNEFNSpQLMC and TPERKTSpVPNNWHC, respectively) were used to detect phosphorylations. A cysteine residue was added to the $\mathrm{C}$ terminus to facilitate conjugation with a carrier protein for greater immunogenicity. To generate antibodies, rabbits were boosted with carrier-conjugated phosphopeptides once per month. Pre-immune serums were collected before boost. Injection was conducted every 4 weeks and blood samples were collected every 2 weeks. Blood samples were incubated at $37^{\circ} \mathrm{C}$ for $30 \mathrm{~min}$, and serum and blood cells were separated via high-speed centrifugation. Clarified serum was incubated at $56^{\circ} \mathrm{C}$ for $30 \mathrm{~min}$ to remove complements. The specificity of antibodies was verified by means of peptide dot blot analysis. Images were captured and quantified by a bioluminescence imaging system (UVP BioSpectrum AC Imagine System, UVP). Uncropped plots are provided in Supplementary Fig. 16.

IP and co-IP. IP of $\mathrm{Cdc13}-\mathrm{Myc}_{9}$ was conducted by $9 \mathrm{E} 10$ monoclonal antibodies (Roche). GST-Est1 and GST-Stn 1 were immunoprecipitated by $2 \mu \mathrm{g}$ of a GST antibody (GE). Immunoprecipitated GST-Est1 and GST-Stn1were detected by the same GST antibody (GE, working condition 1:3,000) and co-immunoprecipitated $\mathrm{HA}_{3}-\mathrm{Cdc} 13$ was detected by an HA antibody (Covance, working condition $1: 2,000)$. Images were captured and quantified by a bioluminescence imaging system (UVP BioSpectrum AC Imagine System, UVP).

Dot blot analysis. A quantity of $50 \mathrm{ng}$ of the phosphorylated or unphosphorylated peptides were spotted on nitrocellulose membranes. Dot blot analysis was conducted by standard protocol using phospho-specific antibodies.

In vitro phosphatase assay. In vitro phosphatase experiments were conducted using affinity-purified TAP-tagged Vps74, Pph3, Pph21 and Pph22 complexes. Recombinant Cdc13 substrates Cdc13(227-277), Cdc13-S249A(227-277) and Cdc13-S255A(227-277) were purified and pre-phosphorylated by immunoprecipitated Mec1 kinase. The kinase assay was initiated in $40 \mu \mathrm{l}$ of kinase buffer by the addition of $10 \mathrm{mCi}\left[\gamma_{-}{ }^{32} \mathrm{P}\right] \mathrm{ATP}\left(3,000 \mathrm{Ci} \mathrm{mmol}^{-1}\right.$, Amersham Pharmacia Biotech), substrate $(2 \mu \mathrm{g}$ of recombinant $\mathrm{Cdc} 13)$ and ATP to $100 \mu \mathrm{M}$ (ref. 22). In vitro phosphatase experiments used affinity-purified, TAP-tagged Pph3, Pph21 or Pph22 complexes. The immunopurified phosphatase ${ }^{64}$ were incubated with recombinant $\mathrm{Cdc} 13$ proteins previously created in vitro by phosphorylating with Mec1 kinase. Sample buffer $(2 \times)$ was added, and reactions were separated by $10 \%$ SDS-PAGE and subjected to autoradiography using an X-ray film. Images were captured and quantified by a bioluminescence imaging system (UVP BioSpectrum AC Imagine System, UVP).

Southern blot analysis and telomere length measurement. When DNA was examined from individual colonies, the colony was expanded in $2 \mathrm{ml}$ of liquid medium to obtain enough DNA for Southern analysis ${ }^{65}$. Genomic DNAs were digested with KpnI, separated by $1.2 \%$ agarose gel electrophoresis, and transferred to Genescreen Plus membrane (PerkinElmer). The blot was probed by a 341-bp ${ }^{32} \mathrm{P}$-labelled (Invitrogen) XhoI-KpnI fragment from the $3^{\prime}$-end of $\mathrm{Y}^{\prime}$ element. Spore cells were serially diluted into or restreaked onto YPD medium. Liquid cultures were generated by inoculating spore colonies from the tetrad plate into $10 \mathrm{ml}$ of liquid YEPD medium. Cultures were diluted repeatedly 1:10,000 into fresh medium at 48 or $72 \mathrm{~h}$ (refs 22,65 ). Genomic DNAs were digested with XhoI, separated by $1 \%$ agarose gel electrophoresis and transferred to Genescreen Plus membrane (PerkinElmer). The blot was probed by a 550-bp ${ }^{32} \mathrm{P}$-labelled (Invitrogen) EcoRI fragment of $\mathrm{TG}_{1-3}$ sequence.

De novo telomere addition assay. De novo telomere addition assay was performed as previously described ${ }^{45}$. UCC5706 strain was kindly provided by $\mathrm{Dr}$ Daniel Gottschling. The pph21::KanMX4 and pph22::KanMX4 mutants were constructed by transforming $p p h 21::$ KanMX 4 and $p p h 22::$ KanMX4 PCR fragments, respectively, into UCC5706. Equal number of cells was collected for DNA preparation, enzyme digestion and Southern blot analysis.

Chromatin IP. ChIP analysis was performed as described ${ }^{25}$. In brief, cells were grown, G1 arrested and released at $24^{\circ} \mathrm{C}$ in YPD broth. After crosslinking in $1 \%$ formaldehyde, cells were lysed and sonicated. IPs were carried out with anti-Myc 9E10 (Roche) and Protein G Dynabeads (Dynal) against C-terminally $\mathrm{Myc}_{18^{-}}$ tagged Est 2 and Est 1 . Both an aliquot of sonicated cleared extract (input) and the immunoprecipitated material were de-crosslinked in Tris/EDTA buffer (TE) plus $1 \%$ SDS for at least $8 \mathrm{~h}$ at $65^{\circ} \mathrm{C}$. Quantification of immunoprecipitated DNA was obtained by real-time PCR using SYBR Green detection (Kappa) on an Applied Biosystems HT7500 machine and software, and was expressed as percent of 
starting (input) material. Primers used in this study are listed in Supplementary Table 4. In a given synchrony, samples from each time point were amplified in duplicate or triplicate to obtain an average value for each sample. In addition, each synchrony was repeated six times; the data are presented as the mean of the three or more synchronies plus or minus s.d.'s. Where applicable, a two-tailed Student's $t$-test was used to determine statistical significance $(P$ value $\leq 0.05)$

Cell cycle analysis of ChIP samples. For ChIP assay, cells were grown at $30^{\circ} \mathrm{C}$ in raffinose and arrested by $\alpha$-factor. Galactose was added for an additional $3 \mathrm{~h}$. Cells were then transferred to glucose medium with $\alpha$-factor for 15 min before the $\alpha$ factor was removed by centrifugation. Cells were released into cell cycle at $24^{\circ} \mathrm{C}$ by the addition of protease. Samples were taken at 0, 45, 60, 70, 80 and $90 \mathrm{~min}$, and were processed for fluorescence-activated cell sorting and ChIP analyses.

MS analysis. In order to identify the phosphorylation sites of $\mathrm{Cdc13}$, Cdc13-Myc, was overexpressed from pYES2-Cdc13-Myc ${ }_{9}$ in nocodazole-arrested yeast, isolated by pull-down and SDS-PAGE and followed by the in-gel enzyme digestion. The tryptic peptides of $\mathrm{Cdc} 13$ were analysed by nanoscale liquid chromatography coupled to tandem mass spectrometry (nano LC-MS/MS) instrument (LTQ-FT, Thermo Fisher Scientific). The MS/MS spectra data were converted as mgf format from experiment RAW file by MM File Conversion Tools ${ }^{66}$ (http:// www.massmatrix.net) then analysed by MassMatrix ${ }^{67}$ for MS/MS ion search. The search parameters in MassMatrix including the error tolerance of precursor ions and the MS/MS fragment ions in spectra were 10 p.p.m. and 0.6 Da. The enzyme for digestion was assigned to be trypsin with the miss cleavage number two. The variable post-translational modifications in search parameters were assigned to include the oxidation of methionine, carbamidomethylation of cysteine and the phosphorylation of serine/threonine/tyrosine. The phosphorylation sites of Cdc13 identified by MS were S306, T308, S314, S324, S333 and S336 (Supplementary Fig. 10).

In vitro kinase assay. For IPs, $50 \mathrm{ml}$ cultures of mid-log-phase cells were collected and lysates were prepared in $500 \mu l$ lysis buffer $(100 \mathrm{mM} \mathrm{NaCl}, 50 \mathrm{mM}$ Tris- $\mathrm{HCl}$, $\mathrm{pH} 7.5,50 \mathrm{mM} \mathrm{NaF}, 50 \mathrm{mM} \beta$-glycerophosphate, $\mathrm{pH}$ 7.4, $2 \mathrm{mM}$ EDTA, $2 \mathrm{mM}$ EGTA, $0.1 \%$ Triton X-100, $1 \mathrm{mM}$ DTT and protease inhibitors ${ }^{68}$. A total of $450 \mu \mathrm{l}$ of supernatant was incubated with $50 \mu \mathrm{l}$ protein G-coated Dynabeads (Invitrogen) and $2 \mu \mathrm{g}$ of M2 anti-Flag antibody (Sigma) for $2 \mathrm{~h}$ at $4{ }^{\circ} \mathrm{C}$. The beads were washed five times with $500 \mu l$ lysis buffer and once with $100 \mu l$ kinase buffer without ATP (50 mM Tris-HCl, pH 7.4, $1 \mathrm{mM}$ DTT, $25 \mathrm{mM} \beta$-glycerophosphate and $5 \mathrm{mM}$ $\mathrm{MgCl}_{2}$ ) and then resuspended in buffer with $10 \mu \mathrm{M}$ ATP, $5 \mu \mathrm{Ci}\left[{ }^{32} \mathrm{P}\right] \mathrm{ATP}$ and $2 \mu \mathrm{g}$ recombinant Cdc13(276-332) proteins for $30 \mathrm{~min}$ at $30^{\circ} \mathrm{C}$. Sample buffer $(2 \times)$ was added, and reactions were separated by $10 \%$ SDS-PAGE and subjected to autoradiography using an X-ray film. Images were captured and quantified by a bioluminescence imaging system (UVP BioSpectrum AC Imagine System, UVP).

Co-IP of TLC1 RNA. For studies of nocodazole-arrested cell cultures, yeast cells were grown at $30^{\circ} \mathrm{C}$ in complete media to $\mathrm{OD}_{660}=0.8$ and treated with nocodazole for $3 \mathrm{~h}$. Extracts were prepared as previously described ${ }^{69}$. One Complete EDTA-free protease inhibitor tablet (Roche) was added for each $10 \mathrm{ml}$ of TMG-50. Typically, $3 \mathrm{mg}$ of total protein was adjusted to $0.5 \%(\mathrm{v} / \mathrm{v})$ Tween-20 and $200 \mathrm{U} \mathrm{ml}^{-1}$ of RNasin (Promega) and RNaseOut (Invitrogen) before our hour incubation at $4{ }^{\circ} \mathrm{C}$ with monoclonal anti-Myc antibody (Roche). Dynabeads Protein G (Invitrogen) equilibrated with TMG-50 plus $0.5 \%(\mathrm{v} / \mathrm{v})$ Tween-20 was then added and incubated for $1 \mathrm{~h}$ at $4{ }^{\circ} \mathrm{C}$. Beads were washed three times with TMG-50 plus $0.5 \%(\mathrm{v} / \mathrm{v})$ Tween-20, once with TMG-50 and resuspended in TMG-50. For the IP of Est1, nocodazole-arrested cells were treated with zymolyase to create spheroplasts, which were then resuspended in $1 \mathrm{ml}$ lysis buffer $(0.4 \mathrm{M}$ sorbitol, $150 \mathrm{mM}$ potassium acetate, $2 \mathrm{mM}$ magnesium acetate, $20 \mathrm{mM}$ HEPES/KOH, $\mathrm{pH} 6.5,100 \mathrm{mg} \mathrm{ml}^{-1}$ phenylmethylsulfonyl fluoride, $1 \mathrm{mg} \mathrm{ml}^{-1}$ pepstatin $\mathrm{A}$ and $0.5 \mathrm{mg} \mathrm{ml}^{-1}$ leupeptin). Spheroplasts were washed three times in lysis buffer, with centrifugation at 4,200 r.p.m. for $3 \mathrm{~min}$ between each wash. Triton X-100 was added to $1 \%$ final concentration and the insoluble fraction was pelleted at 14,000 r.p.m. for $15 \mathrm{~min}$ at $4{ }^{\circ} \mathrm{C}$ (ref. 70). The chromatin pellet was resuspended in $1 \mathrm{ml}$ TMG-50 buffer for further IP. For studies involving synchronous cultures, yeast cultures were grown at $30^{\circ} \mathrm{C}$ in complete media to $\mathrm{OD}_{660}=0.5$. Cultures were then arrested to late $\mathrm{G} 1$ phase by the addition of $0.015 \mu \mathrm{g} \mathrm{ml}^{-1} \alpha$-factor. After arrest, $\alpha$-factor was removed and cells were allowed to progress synchronously through the cell cycle at $24^{\circ} \mathrm{C}$. Samples were taken at $0,45,60,70,80$ and 90 min for fluorescence-activated cell sorting and co-IP analysis. Extracts were prepared for each time point exactly as described. The amount of TLC1 and Actin mRNA copurified were quantified using real-time reverse transcription PCR; Kappa). The enrichment of TLC1 was expressed as the ratio of TLC1 amount co-immunoprecipitated in the presence of anti-Myc 9E10 antibody in samples versus lysate from untagged control strain (background) after normalization to total input (TLC1 test IP/TLC1 test input)(TLC1 no tag input/TLC1 no tag). The enrichment of Actin mRNA was also calculated in the same way and used as a control. The results show an average of three experiments. Statistically significant differences in data sets were established by using a Student's $t$-test. The error bars represent s.d.

\section{References}

1. Zakian, V. A. Structure, function, and replication of Saccharomyces cerevisiae telomeres. Annu. Rev. Genet. 30, 141-172 (1996).

2. McEachern, M. J., Krauskopf, A. \& Blackburn, E. H. Telomeres and their control. Annu. Rev. Genet. 34, 331-358 (2000).

3. Shay, J. W., Zou, Y., Hiyama, E. \& Wright, W. E. Telomerase and cancer. Hum. Mol. Genet. 10, 677-685 (2001).

4. Wellinger, R. J. \& Zakian, V. A. Everything you ever wanted to know about Saccharomyces cerevisiae telomeres: beginning to end. Genetics 191, 1073-1105 (2012).

5. Xu, L., Li, S. \& Stohr, B. A. The role of telomere biology in cancer. Annu. Rev. Pathol. 8, 49-78 (2013).

6. Greider, C. W. \& Blackburn, E. H. Identification of a specific telomere terminal transferase activity in Tetrahymena extracts. Cell 43, 405-413 (1985).

7. Vega, L. R., Mateyak, M. K. \& Zakian, V. A. Getting to the end: telomerase access in yeast and humans. Nat. Rev. Mol. Cell. Biol. 4, 948-959 (2003).

8. Palm, W. \& de Lange, T. How shelterin protects mammalian telomeres. Annu. Rev. Genet. 42, 301-334 (2008).

9. Singer, M. S. \& Gottschling, D. E. TLC1: template RNA component of Saccharomyces cerevisiae telomerase. Science 266, 404-409 (1994).

10. Lendvay, T. S., Morris, D. K., Sah, J., Balasubramanian, B. \& Lundblad, V. Senescence mutants of Saccharomyces cerevisiae with a defect in telomere replication identify three additional EST genes. Genetics 144, 1399-1412 (1996).

11. Counter, C. M., Meyerson, M., Eaton, E. N. \& Weinberg, R. A. The catalytic subunit of yeast telomerase. Proc. Natl Acad. Sci. USA 94, 9202-9207 (1997)

12. Lingner, J. et al. Reverse transcriptase motifs in the catalytic subunit of telomerase. Science 276, 561-567 (1997).

13. Lin, J. J. \& Zakian, V. A. An in vitro assay for Saccharomyces telomerase requires EST1. Cell 81, 1127-1135 (1995).

14. Steiner, B. R., Hidaka, K. \& Futcher, B. Association of the Est1 protein with telomerase activity in yeast. Proc. Natl Acad. Sci. USA 93, 2817-2821 (1996).

15. Virta-Pearlman, V., Morris, D. K. \& Lundblad, V. Est1 has the properties of a single-stranded telomere end-binding protein. Genes Dev. 10, 3094-3104 (1996).

16. Zhou, J., Hidaka, K. \& Futcher, B. The Est1 subunit of yeast telomerase binds the TLC1 telomerase RNA. Mol. Cell. Biol. 20, 1947-1955 (2000).

17. Lin, J. J. \& Zakian, V. A. The Saccharomyces CDC13 protein is a single-strand TG1-3 telomeric DNA-binding protein in vitro that affects telomere behavior in vivo. Proc. Natl Acad. Sci. USA 93, 13760-13765 (1996).

18. Nugent, C. I., Hughes, T. R., Lue, N. F. \& Lundblad, V. Cdc13p: a single-strand telomeric DNA-binding protein with a dual role in yeast telomere maintenance. Science 274, 249-252 (1996)

19. Gao, H., Cervantes, R. B., Mandell, E. K., Otero, J. H. \& Lundblad, V. RPA-like proteins mediate yeast telomere function. Nat. Struct. Mol. Biol. 14, 208-214 (2007).

20. Chandra, A., Hughes, T. R., Nugent, C. I. \& Lundblad, V. Cdc13 both positively and negatively regulates telomere replication. Genes Dev. 15, 404-414 (2001).

21. Nugent, C. I. \& Lundblad, V. The telomerase reverse transcriptase: components and regulation. Genes Dev. 12, 1073-1085 (1998).

22. Tseng, S.-F., Lin, J.-J. \& Teng, S.-C. The telomerase-recruitment domain of the telomere binding protein $\mathrm{Cdc13}$ is regulated by Mec1p/Tel1p-dependent phosphorylation. Nucleic Acids Res. 34, 6327-6336 (2006).

23. Bianchi, A. \& Shore, D. Increased association of telomerase with short telomeres in yeast. Genes Dev. 21, 1726-1730 (2007).

24. Chang, M., Arneric, M. \& Lingner, J. Telomerase repeat addition processivity is increased at critically short telomeres in a Tel1-dependent manner in Saccharomyces cerevisiae. Genes Dev. 21, 2485-2494 (2007).

25. Sabourin, M., Tuzon, C. T. \& Zakian, V. A. Telomerase and Tellp preferentially associate with short telomeres in S. cerevisiae. Mol. Cell. 27, 550-561 (2007).

26. Li, S. et al. Cdk1-dependent phosphorylation of Cdc13 coordinates telomere elongation during cell-cycle progression. Cell 136, 50-61 (2009).

27. Tseng, S.-F., Shen, Z.-J., Tsai, H.-J., Lin, Y.-H. \& Teng, S.-C. Rapid Cdc13 turnover and telomere length homeostasis are controlled by Cdk1-mediated phosphorylation of Cdc13. Nucleic Acids Res. 37, 3602-3611 (2009).

28. Fisher, D., Krasinska, L., Coudreuse, D. \& Novak, B. Phosphorylation network dynamics in the control of cell cycle transitions. J. Cell. Sci. 125, 4703-4711 (2012).

29. Lens, S. M., Voest, E. E. \& Medema, R. H. Shared and separate functions of polo-like kinases and aurora kinases in cancer. Nat. Rev. Cancer. 10, 825-841 (2010).

30. Dephoure, N. et al. A quantitative atlas of mitotic phosphorylation. Proc. Natl Acad. Sci. USA 105, 10762-10767 (2008).

31. Shi, Y. Serine/threonine phosphatases: mechanism through structure. Cell 139, 468-484 (2009).

32. Clarke, P. R., Hoffmann, I., Draetta, G. \& Karsenti, E. Dephosphorylation of Cdc25-C by a type-2A protein phosphatase: specific regulation during the cell cycle in Xenopus egg extracts. Mol. Biol. Cell. 4, 397-411 (1993). 
33. Mueller, P. R., Coleman, T. R. \& Dunphy, W. G. Cell cycle regulation of a Xenopus Weel-like kinase. Mol. Biol. Cell. 6, 119-134 (1995).

34. Castilho, P. V., Williams, B. C., Mochida, S., Zhao, Y. \& Goldberg, M. L. The M phase kinase Greatwall (Gwl) promotes inactivation of PP2A/B55delta, a phosphatase directed against CDK phosphosites. Mol. Biol. Cell. 20, 4777-4789 (2009).

35. Evans, S. K. \& Lundblad, V. Est1 and Cdc13 as comediators of telomerase access. Science 286, 117-120 (1999).

36. Pennock, E., Buckley, K. \& Lundblad, V. Cdc13 delivers separate complexes to the telomere for end protection and replication. Cell 104, 387-396 (2001).

37. Qi, H. \& Zakian, V. A. The Saccharomyces telomere-binding protein Cdc13p interacts with both the catalytic subunit of DNA polymerase alpha and the telomerase-associated Est1 protein. Genes Dev. 14, 1777-1788 (2000).

38. Gao, H. et al. Telomerase recruitment in Saccharomyces cerevisiae is not dependent on Tel1-mediated phosphorylation of Cdc13. Genetics 186, 1147-1159 (2010).

39. Wu, Y. \& Zakian, V. A. The telomeric $\mathrm{Cdc13}$ protein interacts directly with the telomerase subunit Est1 to bring it to telomeric DNA ends in vitro. Proc. Natl Acad. Sci. USA 108, 20362-20369 (2011).

40. Kim, J.-A., Hicks, W. M., Li, J., Tay, S. Y. \& Haber, J. E. Protein phosphatases Pph3, Ptc2, and Ptc3 play redundant roles in DNA double-strand break repair by homologous recombination. Mol. Cell. Biol. 31, 507-516 (2011).

41. Schmitz, K. R. et al. Golgi localization of glycosyltransferases requires a Vps74p oligomer. Dev. Cell 14, 523-534 (2008).

42. Zhang, W. \& Durocher, D. De novo telomere formation is suppressed by the Mec1-dependent inhibition of Cdc13 accumulation at DNA breaks. Genes Dev. 24, 502-515 (2010).

43. Sneddon, A. A., Cohen, P. T. \& Stark, M. J. Saccharomyces cerevisiae protein phosphatase $2 \mathrm{~A}$ performs an essential cellular function and is encoded by two genes. EMBO J. 9, 4339-4346 (1990).

44. Teng, S. C., Chang, J., McCowan, B. \& Zakian, V. A. Telomerase-independent lengthening of yeast telomeres occurs by an abrupt Rad50p-dependent, Rif-inhibited recombinational process. Mol. Cell 6, 947-952 (2000).

45. Diede, S. J. \& Gottschling, D. E. Telomerase-mediated telomere addition in vivo requires DNA primase and DNA polymerases alpha and delta. Cell 99, 723-733 (1999).

46. Hsu, J. Y. et al. Mitotic phosphorylation of histone H3 is governed by Ipl1/ aurora kinase and Glc7/PP1 phosphatase in budding yeast and nematodes. Cell 102, 279-291 (2000).

47. Wang, M. J. et al. Telomere-binding and Stnlp-interacting activities are required for the essential function of Saccharomyces cerevisiae Cdc13p. Nucleic Acids Res. 28, 4733-4741 (2000).

48. DeZwaan, D. C. \& Freeman, B. C. The conserved Estl protein stimulates telomerase DNA extension activity. Proc. Natl Acad. Sci. USA 106, 17337-17342 (2009)

49. Miyoshi, T., Kanoh, J., Saito, M. \& Ishikawa, F. Fission yeast Pot1-Tpp1 protects telomeres and regulates telomere length. Science 320, 1341-1344 (2008).

50. Tomita, K. \& Cooper, J. P. Fission yeast Ccq1 is telomerase recruiter and local checkpoint controller. Genes Dev. 22, 3461-3474 (2008).

51. Yamazaki, H., Tarumoto, Y. \& Ishikawa, F. Tel1(ATM) and Rad3(ATR) phosphorylate the telomere protein Ccq1 to recruit telomerase and elongate telomeres in fission yeast. Genes Dev. 26, 241-246 (2012).

52. Wang, F. et al. The POT1-TPP1 telomere complex is a telomerase processivity factor. Nature 445, 506-510 (2007).

53. Xin, H. et al. TPP1 is a homologue of ciliate TEBP-beta and interacts with POT1 to recruit telomerase. Nature 445, 559-562 (2007).

54. Petersen, P. et al. Protein phosphatase 2A antagonizes ATM and ATR in a Cdk2- and Cdc7-independent DNA damage checkpoint. Mol. Cell. Biol. 26, 1997-2011 (2006).

55. Wu, Y., DiMaggio, Jr P. A., Perlman, D. H., Zakian, V. A. \& Garcia, B. A. Novel phosphorylation sites in the $S$. cerevisiae $\mathrm{Cdc13}$ protein reveal new targets for telomere length regulation. J. Proteome Res. 12, 316-327 (2013)

56. Taggart, A. K., Teng, S. C. \& Zakian, V. A. Est1p as a cell cycle-regulated activator of telomere-bound telomerase. Science 297, 1023-1026 (2002).
57. Baek, G. H., Cheng, H., Kim, I. \& Rao, H. The Cdc48 protein and its cofactor Vms1 are involved in Cdc13 protein degradation. J. Biol. Chem. 287, 26788-26795 (2012).

58. Schulz, V. P. \& Zakian, V. A. The Saccharomyces Pif1 DNA helicase inhibits telomere elongation and de novo telomere formation. Cell 76, 145-155 (1994).

59. Boule, J. B., Vega, L. R. \& Zakian, V. A. The yeast Piflp helicase removes telomerase from telomeric DNA. Nature 438, 57-61 (2005).

60. Rose, M. D., Winston, F. \& Hieter, P. Methods in Yeast Genetics (Cold Spring Harbor Laboratory, 1990).

61. Tsai, Y. L., Tseng, S. F., Chang, S. H., Lin, C. C. \& Teng, S. C. Involvement of replicative polymerases, Tellp, Meclp, Cdc13p, and the Ku complex in telomere-telomere recombination. Mol. Cell. Biol. 22, 5679-5687 (2002).

62. Scherer, S. \& Davis, R. W. Replacement of chromosome segments with altered DNA sequences constructed in vitro. Proc. Natl Acad. Sci. USA 76, 4951-4955 (1979).

63. Tseng, S. F., Chang, C. Y., Wu, K. J. \& Teng, S. C. Importin KPNA2 is required for proper nuclear localization and multiple functions of NBS1. J. Biol. Chem. 280, 39594-39600 (2005)

64. Keogh, M. C. et al. A phosphatase complex that dephosphorylates gammaH2AX regulates DNA damage checkpoint recovery. Nature 439, 497-501 (2006)

65. Teng, S.-C. \& Zakian, V. A. Telomere-telomere recombination is an efficient bypass pathway for telomere maintenance in Saccharomyces cerevisiae. Mol. Cell. Biol. 19, 8083-8093 (1999).

66. Xu, H. \& Freitas, M. A. MassMatrix: a database search program for rapid characterization of proteins and peptides from tandem mass spectrometry data. Proteomics 9, 1548-1555 (2009).

67. Xu, H., Hsu, P. H., Zhang, L., Tsai, M. D. \& Freitas, M. A. Database search algorithm for identification of intact cross-links in proteins and peptides using tandem mass spectrometry. J. Proteome Res. 9, 3384-3393 (2010).

68. Buvelot, S., Tatsutani, S. Y., Vermaak, D. \& Biggins, S. The budding yeast Ipl1/ Aurora protein kinase regulates mitotic spindle disassembly. J. Cell. Biol. 160, 329-339 (2003).

69. Fisher, T. S., Taggart, A. K. P. \& Zakian, V. A. Cell cycle-dependent regulation of yeast telomerase by Ku. Nat. Struct. Mol. Biol. 11, 1198-1205 (2004).

70. Niimi, A., Chambers, A. L., Downs, J. A. \& Lehmann, A. R. A role for chromatin remodellers in replication of damaged DNA. Nucleic Acids Res. 40, 7393-7403 (2012).

\section{Acknowledgements}

We thank Drs Ginger Zakian and Elizabeth Blackburn for their valuable inputs. We also thank Drs Ginger Zakian, Daniel Gottschling, Heidi Feldmann, Clarence S.M. Chan, Jing-Jer Lin, Fang-Jen Lee and Sue Biggins for the gifts of yeast strains. This work was supported by grants from the National Science Council (NSC102-2311-B-002 -044MY3), National Taiwan University (NTU CESRP-101R7602A1) and the National Health Research Institute (NHRI-EX98-9727BI) to S.-C.T.

\section{Author contributions}

S.-C.T. conceived and designed the experiments; Z.-J.S., P.-H.H. and Y.-T.S. performed the experiments with the assistance of C.-W.Y., L.K. and S.-F.T.; M.-D.T. analysed the data; and S.-C.T. wrote the paper with the assistance of Z.-J.S. and M.-D.T.

\section{Additional information}

Supplementary Information accompanies this paper at http://www.nature.com/ naturecommunications

Competing financial interests: The authors declare no competing financial interests.

Reprints and permission information is available online at http://npg.nature.com/ reprintsandpermissions/

How to cite this article: Shen, Z.-J. et al. PP2A and Aurora differentially modify $\mathrm{Cdc13}$ to promote telomerase release from telomeres at G2/M phase. Nat. Commun. 5:5312 doi: 10.1038/ncomms6312 (2014). 


\section{Corrigendum: PP2A and Aurora differentially modify $\mathrm{Cdc} 13$ to promote telomerase release from telomeres at $\mathrm{G} 2 / \mathrm{M}$ phase}

Zih-Jie Shen, Pang-Hung Hsu, Yu-Tai Su, Chia-Wei Yang, Li Kao, Shun-Fu Tseng, Ming-Daw Tsai

\& Shu-Chun Teng

Nature Communications 5:5312 doi: 10.1038/ncomms6312 (2014); Published 12 Nov 2014; Updated 10 Jul 2015

This Article incorrectly cites ref. 51 at the end of the penultimate sentence of the first paragraph of the Discussion: 'Consistently, ataxia telangiectasia mutated (ATM)/ATM- and Rad3-related (ATR)-promoted Ccq1-Est1 interaction was recently revealed ${ }^{51}$. The correct citation is as follows:

Moser, B. A., Chang, Y. T., Kosti, J. \& Nakamura, T. M. Tel1 ${ }^{\text {ATM }}$ and Rad3 ${ }^{\text {ATR }}$ kinases promote Ccq1-Est1 interaction to maintain telomeres in fission yeast. Nat. Struct. Mol. Biol. 18, 1408-1413 (2011). 